\title{
Offline Signature Verification: An Approach Based on Score Level Fusion
}

\author{
H.N. Prakash \\ Department of Studies in Computer Science, \\ University of Mysore, \\ Manasagangothri, Mysore-570 006, India
}

\author{
D. S. Guru \\ Department of Studies in Computer Science \\ University of Mysore \\ Manasagangothri, Mysore-570 006, India
}

\begin{abstract}
In this paper, we propose a new approach for offline signature verification based on score level fusion of distance and orientation features of centroids. The proposed method employs symbolic representation of offline signatures using bi-interval valued feature vector. Distance and orientation features of centroids of offline signatures are used to form bi-interval valued symbolic feature vector for representing signatures. A method of offline signature verification based on the bi-interval valued symbolic representation is presented. Several experiments are conducted on MCYT_ signature database [1] of 2250 signatures to demonstrate the efficacy of the proposed approach based score level fusion for offline signature verification.
\end{abstract}

\section{Keywords}

Offline signature verification; Distance and orientation features; Score level fusion; Bi-interval valued symbolic feature vector: Geometric centroids.

\section{INTRODUCTION}

For signature verification many features are extracted so far by the geometric analysis the signature. The most commonly used features are signature image area, signature height and width, height to width ratio, number of salient points (viz. maxima and minima) and number of characteristic points (viz. cross points and split points) [2]. In addition, direction based features, slant-based features, orientation based features, contour based features, grid based features, texture based features and spectrum based features [2] are also commonly used for signature verification.

In verification, the authenticity of a test signature is evaluated by matching its features against those stored in the knowledgebase. For matching various pattern recognition strategies like Neural Networks [3], Time Warping [4], Hidden Markov Model (HMM) [5] and Support Vector Machine (SVM) [5] have been employed. Symbolic data [6] appear in the form of continuous ratio, discrete absolute interval and multi-valued, multi-valued with weightage, quantitative, categorical, etc. The concept of symbolic data analysis has been extensively studied in the field of cluster analysis and it has been proved both theoretically and experimentally that the clustering approaches based on symbolic data outperform conventional clustering techniques [6]. Recently, a symbolic representation model for 2D shapes has been proposed and it has also shown that symbolic representation model effectively captures shape information [7]. In previous work, we have proposed relative centroid orientations for offline signature verification [8]. Recently, we have proposed relative distances between geometric centroids for offline signature verification [9]. In this paper, bi-interval valued symbolic representation for offline signatures and score level fusion of distances between geometric centroids and corresponding orientations of geometric centroids for signature verification are proposed. The main motivation for our biinterval representation based fusion approach is that the fusion techniques [10] and the symbolic representation of signature in our previous work [11] resulted in good performance in case of online signature verification. In this work, the distances between geometric centroids and the corresponding orientations of geometric centroids are used to form bi-interval symbolic representation. A method of signature verification based on biinterval valued symbolic representation is also proposed.

The rest of the paper is structured as follows: In section 2, extraction of features, method of symbolic representation and verification of offline signatures are presented. In section 3 , the details of the experimentations and the results are summarized. Comparison with other methods is made in section 4. Finally, the conclusions are drawn in section 5 .

\section{PROPOSED METHOD}

In this section, the proposed method feature extraction, biinterval valued symbolic representation of offline signature and further, the signature verification are presented. 


\subsection{Feature Extraction}

The geometric centroids represent the pixel distribution of the signature image which in turn depends on handwritten signature pattern. In the proposed method signature image is binarized using the histogram based global threshold [12]. Then, we find the geometric centroid of the image and subsequently we split the signature image vertically at the geometric centroid to get two partitions. In the next step, we find the geometric centroid of each partition to split each of the partitions horizontally at their geometric centroids. This procedure of finding centroids and splitting the partitions vertically and horizontally at the centroids is continued recursively in an alternative way till a desired depth of the splitting is reached [8], [9]. Generally, we extract $n=$ [(2) ${ }^{r}$-1] centroids, where $r=1,2,3, \ldots, k$. is the number of splits. Centroids extracted for each split portions are labeled as 1, 2, $3, \ldots, n$ in sequence as shown in Figure.1.

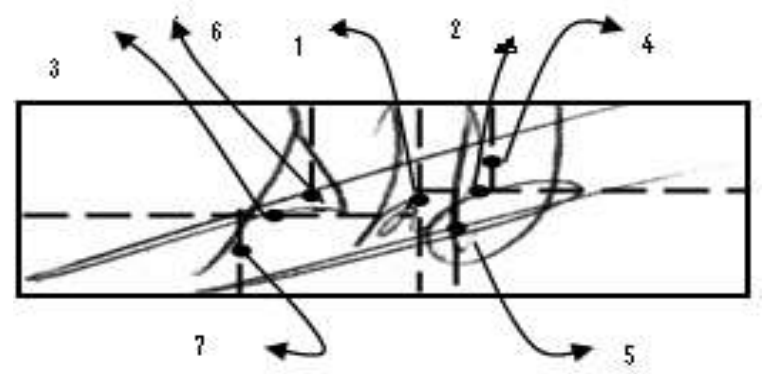

Figure 1. Extraction of geometric centroids of a signature image

A graph of edges joining ' $n$ ' geometric centroids is envisaged in Figure. 2. Let the first geometric centroids be labeled as ' 1 ' and the second as ' 2 ' and so on and so forth until ' $n$ ', the last geometric point. We illustrate the proposed methodology with $n$ $=5$ geometric centroids (corresponding to centroids 1 to 5 ). Each edge is now characterized by two features: length of the edge (which is the distance between geometric centroids) and slope of the edge (which is the orientation of centroids).

A vector $F$ consisting of the lengths of all the edges and corresponding orientations form the symbolic representation of a signature and is given by

$$
\begin{array}{r}
F=\left\{\left(d_{12} \theta_{12}\right),\left(d_{13} \theta_{13}\right), \ldots,\left(d_{1 n} \theta_{1 n}\right),\left(d_{23} \theta_{23}\right),\left(d_{24} \theta_{24}\right), \ldots,\right. \\
\left.\left(d_{i j} \theta_{i j}\right), \ldots,\left(d_{n-1 n} \theta_{n-1 n}\right)\right\}
\end{array}
$$

where $d_{i j}$ is the distance (length) of the edge directed from node $i$ to node $j$, and $\theta_{i j}$ is the orientation of the edge directed from node $i$ to node $j$, for $1 \leq i \leq n-1, \quad 2 \leq j \leq n$, and $i<j$.

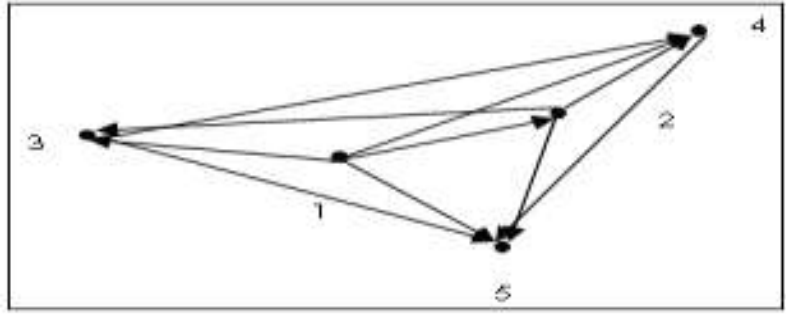

Figure 2 Geometric center points with labels as nodes and the corresponding edges

For $n$ geometric centroids we get $(n(n-1)) / 2$ distances and $(n(n-$ 1)) $/ 2$ orientations. Say for $n$ centroids we get $m=(n(n-1)) / 2$ features, and then the above Eq. (1) can be represented by

$$
\begin{array}{r}
F=\left\{\left[d_{1}, \theta_{1}\right],\left[d_{2}, \theta_{2}\right],\left[d_{3}, \theta_{3}\right], \ldots,\left[d_{k-1}, \theta_{k-1}\right],\left[d_{k}, \theta_{k}\right],\right. \\
\left.\left[d_{k+1}, \theta_{k+1}\right], \ldots,\left[d_{m}, \theta_{m}\right]\right\}
\end{array}
$$

\subsection{Symbolic Representation of Signature}

Recently, on-line signature verification model based on symbolic representation using global features has been proposed [6] and this model has shown a good verification performance. In the present work, we use both relative distances and orientations features for symbolic representation of offline signatures and we introduce score level fusion of distance and orientation features for offline signatures verification.

Let $\left[S_{1}, S_{2}, S_{3}, \ldots, S_{n}\right]$ be a set of $n$ samples of a signature class say $C_{j} ; j=1,2,3, \ldots, N$ ( $N$ denotes the number of individuals) and let $F_{i j}=\left\{\left[d_{i 1}, \theta_{i 1}\right],\left[d_{i 2}, \theta_{i 2}\right], \ldots,\left[d_{i m}, \theta_{m}\right]\right\}$ be the vector of $m$ bi-valued features characterizing the signature sample $S_{i}$ of the class $C_{j}$. Let $\overline{D_{j k}} ; k=1,2, \ldots, m$ and $\bar{\phi}_{j k} ; k=$ $1,2, \ldots, m$ be the means of the $k^{\text {th }}$ distance feature values and the $k^{\text {th }}$ orientation feature values respectively obtained from all the $n$ samples of the class $C_{j}$. i.e.,

$$
\bar{D}_{j k}=\frac{1}{n} \sum_{i=1}^{n} d_{i k} \text { and } \bar{\phi}_{j k}=\frac{1}{n} \sum_{i=1}^{n} \theta_{i k}
$$

Similarly, let $\sigma_{k}^{d}$ and $\sigma_{k}^{\theta}$ be the standard deviations of the $k^{\text {th }}$ distance feature values and the $k^{\text {th }}$ orientation feature values obtained from all the $n$ samples i.e.,

$$
\begin{aligned}
& \sigma_{j k}^{d}=\left[\frac{1}{n} \sum_{i=1}^{n}\left(d_{i k}-\bar{D}_{j k}\right)^{2}\right]^{\frac{1}{2}} \text { and } \\
& \sigma_{j k}^{\theta}=\left[\frac{1}{n} \sum_{i}^{n}\left(\theta_{i k}-\bar{\phi}_{j k}\right)^{2}\right]^{\frac{1}{2}}
\end{aligned}
$$


We compute the means $\bar{D}_{k}$ and $\bar{\phi}_{k}$, and the standard deviations $\sigma_{k}^{d}$ and $\sigma_{k}^{\theta} \quad(k=1,2,3, \ldots, m)$ for all the distance and orientation features respectively for a signature class. Now, we recommend capturing variations in each feature value in the form of bi-interval $\left(\left[d_{j k}^{-}, d_{j k}^{+}\right],\left[\theta_{j k}^{-}, \theta_{j k}^{+}\right]\right)$.

where $d_{j k}^{-}=\bar{D}_{j k}-\alpha \sigma_{j k}^{d}$ and $d_{j k}^{+}=\bar{D}_{j k}+\alpha \sigma_{j k}^{d}$

$$
\text { and } \theta_{j k}^{-}=\bar{\phi}_{j k}-\alpha \sigma_{j k}^{\theta} \text { and } \theta_{j k}^{+}=\bar{\phi}_{j k}+\alpha \sigma_{j k}^{\theta}
$$

Here $\alpha$, is a parameter to fix up feature dependent threshold and hence to obtain variable width interval representation for each feature.

A reference signature representing the entire $j^{\text {th }}$ class (all samples of a person) is formed by the use of bi-interval type data vector $R F_{j}$ consisting of the distances (lengths) and corresponding orientations/slopes of all the possible edges which form the symbolic representation of a signature and is given by

$$
\begin{array}{r}
R F_{j}=\left\{\left(\left[d_{j 1}^{-}, d_{j 1}^{+}\right],\left[\theta_{j 1}^{-}, \theta_{j 1}^{+}\right]\right),\left(\left[d_{j 2}^{-}, d_{j 2}^{+}\right],\left[\theta_{j 2}^{-}, \theta_{j 2}^{+}\right]\right), \ldots,\right. \\
\left.\left(\left[d_{j m}^{-}, d_{j m}^{+}\right],\left[\theta_{j m}^{-}, \theta_{j m}^{+}\right]\right)\right\}
\end{array}
$$

where $m=n(n-1) / 2$ corresponding to number of edges.

It shall be noted that unlike conventional feature vector, this is a vector of bi-interval valued features and this symbolic feature vector is stored in the knowledge base as a representative of the signature class. Thus, the knowledgebase has $N$ number of symbolic vectors because of $N$ individuals.

\subsection{Signature Verification}

The signature verification technique proposed in this work considers a query signature, which is described by a set of $m$ bivalued features of type crisp corresponding to distance and orientation features and compares it with the bi-interval type feature values of the claimed identity (reference signature) in the knowledgebase. Let

$$
F_{Q}=\left\{\left[d_{t 1}, \theta_{t 1}\right],\left[d_{t 2}, \theta_{t 2}\right],\left[d_{t 3}, \theta_{t 3}\right], \ldots,\left[d_{t m}, \theta_{t m}\right]\right\}
$$

be the query signature described by $m$ dimensional bi-valued feature vector. Let $R F_{R}$ (Eq. (6)) be the reference signature of the claimed identity described by bi-interval-valued feature vector

Each $k^{\text {th }}$ distance feature value and corresponding orientation features of the test signature is compared with the corresponding intervals in $R F_{R}$ to examine whether the test signature feature values lies within the corresponding intervals. The number of features of a test signature, which fall inside the corresponding intervals of the respective reference signature, is defined to be the degree of authenticity.
Further, we define $A_{c}^{d}$ the acceptance count (matching score) for distance features and $A_{c}^{\theta}$ acceptance count (matching score) for orientation features as follows

$$
A_{c}^{d}=\sum_{k=1}^{m} C\left(d_{t k},\left[d_{j k}^{-}, d_{j k}^{+}\right]\right)
$$

where,

$C\left(d_{t k},\left[d_{j k}^{-}, d_{j k}^{+}\right]\right)= \begin{cases}1 & \text { if }\left(d_{t k} \geq d_{j k}^{-} \text {and } d_{t k} \leq d_{j k}^{+}\right) \\ 0 & \text { otherwise }\end{cases}$

and

$$
A_{c}^{\theta}=\sum_{k=1}^{m} C\left(\theta_{t k},\left[\theta_{j k}^{-}, \theta_{j k}^{+}\right]\right)
$$

where,

$C\left(\theta_{t k},\left[\theta_{j k}^{-}, \theta_{j k}^{+}\right]\right)= \begin{cases}1 & \text { if }\left(\theta_{t k} \geq \theta_{j k}^{-} \text {and } \theta_{t k} \leq \theta_{j k}^{+}\right) \\ 0 & \text { otherwise }\end{cases}$

\section{Score level fusion strategies for signature verification}

Information fusion in signature verification system (biometrics) could be at the following fusion levels:

- Sensor level fusion refers to the combination of raw data from the sensors which acquire data.

- Feature level fusion refers to the combination of different feature vectors obtained by feature extraction algorithm to the same raw data.

- Score level fusion refers to the combination of matching scores

- Decision level fusion refers to the combination of decisions already taken by the individual systems

More commonly used fusion is score level fusion in biometrics. We adopt score level fusion in this work. For the score level fusion ("Max" / "Mean") algorithms, we define separately an acceptance count $A_{c}$ for the test signature to decide if signature is authentic is as follows. An acceptance count is nothing but matching score obtained by comparing the query feature with that of reference

\section{"Max" Algorithm}

$$
A_{c}=\max \left(A_{c}^{d}, A_{c}^{\theta}\right)
$$

In this case maximum of $\left(A_{c}^{d}, A_{c}^{\theta}\right)$ is used as acceptance count for the system. If this acceptance count for a test signature is greater than the predefined threshold $(T)$ then test signature is considered to be genuine. 
"Mean" Algorithm

$$
A_{c}=\operatorname{avg}\left(A_{c}^{d}, A_{c}^{\theta}\right)
$$

In this case average of $\left(A_{c}^{d}, A_{c}^{\theta}\right)$ is used as acceptance count for the system. If this acceptance count for a test signature is greater than the predefined threshold $(T)$ then test signature is considered to be genuine.

Now, we define the total acceptance count $A_{c}^{t}$ as follows

$$
A_{c}^{t}=\beta A_{c}^{d}+\lambda A_{c}^{\theta}
$$

Where $\beta$ and $\lambda$ are weightage factors. The above total acceptance count could be calculated strictly for two cases: 1 ) $\beta=1$ and $\lambda=0$ considering only distance features and with

2) $\beta=0$ and $\lambda=1$ considering only orientation features for verification purpose. If the total acceptance count is greater than the predefined threshold $(T)$ then the test signature is considered as genuine otherwise as forgery.

For each fusion method we separately define $A_{c}$. If the corresponding acceptance count for a test signature is greater than the predefined threshold $(T)$ then test signature is considered to be genuine. The operating point for our experimentation is set by empirically fixing up the values for $T$ and $\alpha$ [6]. For decision scheme a single threshold or multiple threshold related to different identities could be used. We have empirically set threshold $T=m^{*} 0.55$ and $\alpha=1$ (for logical Max algorithm) and $T=m / 2$ and $\alpha=1$ (for Mean algorithm) as common threshold.

\section{EXPERIMENTATION AND RESULTS}

The dataset: The MCYT-75 offline signature corpus [1] consists of 2250 signatures from 75 individuals. Each individual class consist 30 signatures; out of which 15 are genuine and remaining 15 are skilled forgeries. Totally it forms a signature database of 1125 (i.e. $75 \times 15$ ) genuine and 1125 (i.e. $75 \times 15$ ) forged offline signatures. See Figure 3.

Experimental Setup: The MCYT_signature subcorpus is split into training and testing sets. We trained the system with training set of 5, 7 and 9 genuine signatures of each individual selected randomly. The test set consists of the remaining samples of genuine signatures and all the forgery signatures. Our procedure is similar to the international signature verification competition SVC 2004. We have used normalized distances and orientations features for our experimentations. For evaluation of the proposed method for verification performance, in this work we adopt AER (Average Error Rate), which is average of FAR (False Acceptance Rate) and FRR (False Rejection Rate).
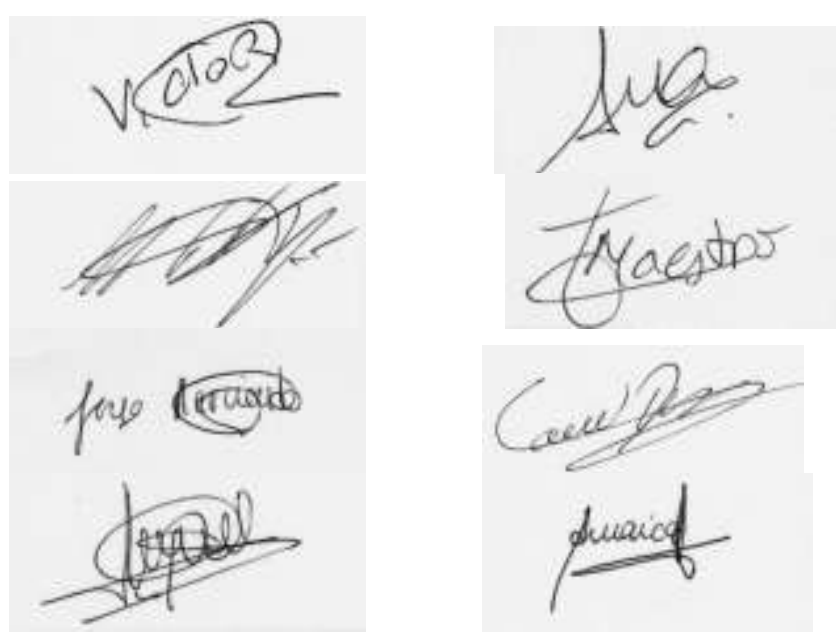

Figure 3. Samples signatures from MCYT_ signature corpus

\subsection{Results Based on Only Distance Features}

The results of experimentations using only the distance features ( $\beta=1$ and $\lambda=0$ in Eq. 12) are tabulated in this subsection. The variations of FAR and FRR for various training samples and under varying number of geometric centroids are given in Tables 1-3. We measure the performance in terms of commonly used average error rate (AER).

Table 1. Verification performances (Average error rates) for 31 centroids, Threshold $=\mathbf{2 3 3}$

\begin{tabular}{|c|c|c|c|}
\hline $\begin{array}{c}\text { Training Samples } \\
\text { per Class }\end{array}$ & FRR & FAR & AER \\
\hline $\mathbf{5}$ & 42.53 & 19.82 & 30.50 \\
\hline $\mathbf{7}$ & 32.83 & 24.04 & 28.31 \\
\hline $\mathbf{9}$ & 27.77 & 26.11 & 26.90 \\
\hline
\end{tabular}

ITable 2. Verification performances (Average error rates) for 63 centroids, Threshold $=977$

\begin{tabular}{|c|c|c|c|}
\hline $\begin{array}{c}\text { Training Samples } \\
\text { Per Class }\end{array}$ & FRR & FAR & AER \\
\hline $\mathbf{5}$ & 37.20 & 20.26 & 28.73 \\
\hline $\mathbf{7}$ & 26.16 & 26.13 & 26.10 \\
\hline $\mathbf{9}$ & 20.22 & 29.51 & 24.86 \\
\hline
\end{tabular}


Table 3. Verification performances (Average error rates) for 127 centroids, Threshold $=\mathbf{4 0 0 1}$

\begin{tabular}{|c|c|c|c|}
\hline $\begin{array}{c}\text { Training Samples } \\
\text { per Class }\end{array}$ & FRR & FAR & AER \\
\hline $\mathbf{5}$ & 37.20 & 21.06 & 28.23 \\
\hline $\mathbf{7}$ & 22.83 & 26.57 & 24.12 \\
\hline $\mathbf{9}$ & 19.11 & 24.11 & $\mathbf{2 1 . 6 1}$ \\
\hline
\end{tabular}

\subsection{Results Based on Only Orientation Features}

The results of experimentations using only the orientations features ( $\beta=0$ and $\lambda=1$ in Eq. 12) are tabulated in this subsection. The variations of FAR, FRR and AER for various training samples and under varying number of geometric centroids are given in Tables 4-6.

Table 4 .Verification performances (AER) for 31 centroids, Threshold $=\mathbf{2 3 3}$

\begin{tabular}{|c|c|c|c|}
\hline $\begin{array}{c}\text { Training Samples } \\
\text { per Class }\end{array}$ & FRR & FAR & AER \\
\hline $\mathbf{5}$ & 42.13 & 16.08 & 29.10 \\
\hline $\mathbf{7}$ & 26.00 & 23.37 & 24.68 \\
\hline $\mathbf{9}$ & 22.44 & 24.08 & 23.26 \\
\hline
\end{tabular}

Table 5. Verification performances (AER) for 63 centroids, Threshold $=977$

\begin{tabular}{|c|c|c|c|}
\hline $\begin{array}{c}\text { Training Samples } \\
\text { per Class }\end{array}$ & FRR & FAR & AER \\
\hline $\mathbf{5}$ & 32.26 & 20.88 & 26.57 \\
\hline $\mathbf{7}$ & 18.33 & 28.80 & 23.56 \\
\hline $\mathbf{9}$ & 15.11 & 28.80 & 21.95 \\
\hline
\end{tabular}

Table 6. Verification performances (AER) for 127 centroids, Threshold $=4001$

\begin{tabular}{|c|c|c|c|}
\hline $\begin{array}{c}\text { Training Samples } \\
\text { per Class }\end{array}$ & FRR & FAR & AER \\
\hline $\mathbf{5}$ & 34.50 & 18.13 & 26.31 \\
\hline $\mathbf{7}$ & 18.42 & 25.51 & 21.76 \\
\hline $\mathbf{9}$ & 14.66 & 25.11 & $\mathbf{1 9 . 8 8}$ \\
\hline
\end{tabular}

\subsection{Results based on Score level Fusion of Distance and Orientation Features}

"Max" Algorithm: The results of experimentations using

"Max" algorithm (Eq.10) and using both the distance and orientation features are tabulated in this subsection. The variations of FAR, FRR and AER for various training samples and for varying number of geometric centroids are given in Tables 7-9.

Table7. Verification performances (AER) using "Max" algorithm for 31 centroids, Threshold $=233$

\begin{tabular}{|c|c|c|c|}
\hline \multirow{2}{*}{$\begin{array}{c}\text { Training } \\
\text { Samples }\end{array}$} & \multicolumn{3}{|c|}{ "Max" algorithm } \\
\cline { 2 - 4 } & FRR & FAR & AER \\
\hline $\mathbf{5}$ & 39.49 & 20.88 & 30.18 \\
\hline $\mathbf{7}$ & 33.54 & 18.21 & 25.87 \\
\hline $\mathbf{9}$ & 28.33 & 17.19 & 22.76 \\
\hline
\end{tabular}

Table 8. Verification performances (AER) using "Max" algorithm for 63 centroids, Threshold $=976$

\begin{tabular}{|c|c|c|c|}
\hline \multirow{2}{*}{$\begin{array}{c}\text { Training } \\
\text { Samples }\end{array}$} & \multicolumn{3}{|c|}{ "Max" algorithm } \\
\cline { 2 - 4 } & FRR & FAR & AER \\
\hline $\mathbf{5}$ & 31.21 & 19.41 & 25.31 \\
\hline $\mathbf{7}$ & 24.71 & 19.53 & 22.12 \\
\hline $\mathbf{9}$ & 19.83 & 18.85 & 19.34 \\
\hline
\end{tabular}

Table 9. Verification performances (AER) using "Max" algorithm for 127 centroids, Threshold $=4001$

\begin{tabular}{|c|c|c|c|}
\hline \multirow{2}{*}{$\begin{array}{c}\text { Training } \\
\text { Samples }\end{array}$} & \multicolumn{3}{|c|}{ "Max" algorithm } \\
\cline { 2 - 4 } & FRR & FAR & AER \\
\hline $\mathbf{5}$ & 30.11 & 19.75 & 24.93 \\
\hline $\mathbf{7}$ & 20.22 & 19.41 & 19.81 \\
\hline $\mathbf{9}$ & 17.11 & 19.41 & $\mathbf{1 8 . 2 6}$ \\
\hline
\end{tabular}

"Mean" Algorithm: The results of experimentations using "Mean" algorithm (Eq.11) and using both the distance and orientation features are tabulated in this subsection. The variations of FAR, FRR and AER for various training samples and for varying number of geometric centroids are given in Tables 10-12.

Table 10.Verification performances (AER) using "Mean" algorithm for 31 centroids, Threshold $=\mathbf{2 3 3}$

\begin{tabular}{|c|c|c|c|}
\hline \multirow{2}{*}{$\begin{array}{c}\text { Training } \\
\text { Samples }\end{array}$} & \multicolumn{3}{|c|}{ "Mean" algorithm } \\
\cline { 2 - 4 } & FRR & FAR & AER \\
\hline $\mathbf{5}$ & 35.41 & 23.44 & 29.42 \\
\hline $\mathbf{7}$ & 26.12 & 21.44 & 23.78 \\
\hline
\end{tabular}




\begin{tabular}{|l|l|l|l|}
\hline $\mathbf{9}$ & 22.61 & 20.22 & 21.41 \\
\hline
\end{tabular}

Table 11. Verification performances (AER) using "Mean" algorithm for 63 centroids, Threshold $=976$

\begin{tabular}{|c|c|c|c|}
\hline \multirow{2}{*}{$\begin{array}{c}\text { Training } \\
\text { Samples }\end{array}$} & \multicolumn{3}{|c|}{ "Mean" algorithm } \\
\cline { 2 - 4 } & FRR & FAR & AER \\
\hline $\mathbf{5}$ & 29.23 & 17.40 & 23.31 \\
\hline $\mathbf{7}$ & 24.11 & 16.82 & 20.46 \\
\hline $\mathbf{9}$ & 15.41 & 21.81 & 18.61 \\
\hline
\end{tabular}

Table 12. Verification performances (AER) using "Mean"“ algorithm for 127 centroids, Threshold $=4001$

\begin{tabular}{|c|c|c|c|}
\hline \multirow{2}{*}{$\begin{array}{c}\text { Training } \\
\text { Samples }\end{array}$} & \multicolumn{3}{|c|}{ "Mean" algorithm } \\
\cline { 2 - 4 } & FRR & FAR & AER \\
\hline $\mathbf{5}$ & 29.40 & 18.11 & 23.75 \\
\hline $\mathbf{7}$ & 18.33 & 20.00 & 19.16 \\
\hline $\mathbf{9}$ & 14.85 & 19.82 & $\mathbf{1 7 . 3 3}$ \\
\hline
\end{tabular}

Table 13. Comparison of best verification performances

\begin{tabular}{|l|c|c|c|}
\hline Methods & FRR & FAR & AER \\
\hline Distance based & 19.11 & 24.11 & $\mathbf{2 1 . 6 1}$ \\
\hline Orientation based & 14.66 & 25.11 & $\mathbf{1 9 . 8 8}$ \\
\hline Fusion: "Max" & 17.11 & 19.41 & $\mathbf{1 8 . 2 6}$ \\
\hline Fusion: "Mean" & 14.85 & 19.82 & $\mathbf{1 7 . 3 3}$ \\
\hline
\end{tabular}

Comparison of the best results: On comparison of results tabulated in tables 1-12, the proposed fusion approach gives the good results for 127 centroids for 9 training samples. Further, the verification results of fusion approaches are better than that of the approaches which use only distance features or only orientation features. The best results obtained for different methods are tabulated Table 13. On comparison of the results in Table 13 "Mean" fusion shows the best performance.

\section{COMPARISON WITH OTHER METHODS}

It is very difficult to compare the performances of different signature verification systems because different systems use different signature databases. Hence here we list the performances of different systems and our system with respect to size of database and the number of writers. From the comparison (see Table 14) it is clear with the large database size the proposed system yields lower AER (17.33) and hence the performance of the system is encouraging. In literature, an other model which makes use of centroids as features is reported in [17]. However, it employs directly the Euclidean distance between the centroids of a test signature and that of the stored signature and hence it is not invariant to scaling. Thus, the performance is reported only on a small database of their own. So, we feel it is not required to consider for comparative study.

Table 14. Comparison with other methods

\begin{tabular}{|l|c|c|c|}
\hline Similar works & $\begin{array}{c}\text { No. of } \\
\text { Writers }\end{array}$ & $\begin{array}{c}\text { Database } \\
\text { Size }\end{array}$ & $\begin{array}{l}\text { AER } \\
(\mathbf{\%})\end{array}$ \\
\hline $\begin{array}{l}\text { 1) Proposed methods } \\
\text { a) "MAX" fusion } \\
\text { b) "MEAN" fusion }\end{array}$ & $\mathbf{7 5}$ & $\mathbf{2 2 5 0}$ & $\mathbf{1 8 . 2 6}$ \\
$\mathbf{2 2 5 0}$ & $\mathbf{1 7 . 3 3}$ \\
\hline $\begin{array}{l}\text { 2) Meenakshi K. K } \\
\text { et., al [13] }\end{array}$ & 55 & 1320 & 21.9 \\
\hline $\begin{array}{l}\text { 3) Shankar A. P. and } \\
\text { Rajagopalan [4] }\end{array}$ & 100 & 1431 & 35.0 \\
\hline $\begin{array}{l}\text { 4) Srihari et., al [14] } \\
\text { a) Distance Threshold } \\
\text { (GSC) } \\
\text { b) Distance statistics } \\
\text { c) Naïve Bayes } \\
\text { d) One Class- SVM }\end{array}$ & 55 & 1320 & 22.4 \\
\hline $\begin{array}{l}\text { 5) Fang B. and Y. Y. } \\
\text { Tang [15] }\end{array}$ & 55 & 1320 & 23.4 \\
\hline $\begin{array}{l}\text { 6) Fang B. et. al[16] } \\
\text { (a) 2D elastic } \\
\text { matching } \\
\text { (b) Horizontal and } \\
\text { vertical projections } \\
\text { (c) Global shape } \\
\text { features }\end{array}$ & 55 & 1320 & 22.0 \\
\hline
\end{tabular}

\section{CONCLUSION}

In this paper, we have proposed a score level fusion method for offline signature verification. The verification method proposed is based on proposed bi-interval valued symbolic representation of signature using relative distances and relative orientations of geometric centroids as features. The main finding of this work is that offline signature verification based on proposed fusion approach achieves further reduction in AER. The proposed approach shows the lower AER (AER = 17.33 for "MEAN" fusion and $\mathrm{AER}=18.26$ for "MAX" fusion) than the approaches which directly use either distance features or orientation features. We have made a successful attempt to achieve reduction in AER by exploring the applicability of fusion method for offline signature verification by using bi-interval valued feature vector representation of signature and symbolic data concepts. The proposed method is very simple compared to methods which employ support vector machines (SVMs), Hidden Markov models (HMMs) and Neural Networks (NNs) which are computationally intensive for signature verification. Further, the results obtained by the proposed method as a stand- 
alone approach are very impressive compared to many other existing stand-alone approaches of verification found in the literature.

\section{ACKNOWLEDGMENTS}

Authors thank Dr. Julian Firrez Auguilar, Biometric Research Lab-AVTS, Madrid, Spain for providing MCYT_signature dataset.

\section{References}

[1] Ortega-Garcia J., J. Fierrez-Aguliar and D. Simon, 2003. MCYT baseline corpus: A bimodal database. IEE proceedings Vision, Image and Signal Processing, pp. 395401.

12] Dimauro G., S. Impedovo, M.G. Lucchese, R. Modugno and G. Pirlo, 2004. Recent advancement in automatic signature verification. Proceedings of $9^{\text {th }}$ International Workshop on Frontiers in Handwriting Recognition (IWFHR), pp. 179-184.

[3] Bajaj R and Chaudhary S., 1997. Signature verification using multiple neural classifiers. Pattern Recognition, vol. 30 , pp. $1-87$.

[4] Shankar A.P and A. N. Rajagopalan, 2007. Offline signature verification using DTW. Pattern Recognition Letters, vol. 28, pp. 1407-1414.

[5] Justino E.J.R, F. Bortolazzi and R. Sabourin. 2005. A comparison of SVM and HMM classifiers in the offline signature verifications. Pattern Recognition Letters, vol.26. issue 9, pp.1377-1385.

[6] Bock H. H. and Diday E., 1999. Analysis of symbolic data, Springer Verilag publication.

[7] Guru D.S. and H. S. Nagendraswamy, 2006. Symbolic representation of two-dimensional shapes. Pattern Recognition Letters, vol.28, pp.144-155.

[8] Prakash H.N. and D.S. Guru. 2009. Relative orientations of Geometric Centroids for Offline Signature Verification, International conference on advanced pattern recognition, (ICAPR-2009), ISI, Kolkata, India, pp. 201- 204.

[9] Prakash H.N. and D.S. Guru, 2009. Geometric Centroids and their Relative Distances for Offline Signature Verification. International Conference Document Analysis and Recognition, (ICDAR-2009), Barcelona, Spain. IEEE, 121-125.
[10] Aguilar J. F., Krawczyk S, Garcia J. O. and Anil k. Jain, 2005. Fusion of Local and Regional Approaches for On-line Signature Verification, International Workshop on Biometric Recognition System (IWBRS), LNCS 3781, pp.188-196, Springer Verlag. Berlin, Heidelberg.

[11] Guru D.S. and H.N. Prakash, 2009. On-line signature verification and recognition: An approach based on symbolic representation. IEEE transactions on pattern analysis and machine intelligence, vol. 31, no. 6, pp.10591073.

[12] Otsu N., 1994, A threshold selection method from grey level histogram. IEEE Transactions on Systems, Man and Cybernetics, vol. 9, pp.62-66.

[13] Kalera M.K., Surgur Srihari and Aihua Xu, 2004. Offline Signature verification and identification using distance statistics. International Journal of Pattern Recognition and Artificial Intelligence. vol. 18, no.7, pp. 1339-1360.

[14] Srihari S.N., Aihua Xu and M.K. Kalera, 2004. Learning strategies and classification methods for offline signature verification. Proceedings of $6^{\text {th }}$ International Workshop on Frontiers in Handwriting Recognition (IWFHR), Tokyo, Japan ,2004, IEEE, Computer society Press, pp. 161-166.

[15] Fang B. and Yaun Yan Tang, 2005. Improved class statistics estimation for sparse data Problems in Offline signature verification. IEEE Transactions on Systems, Man and Cybernetics, vol. 35, no. 3, pp.276-286.

[16] Fang B., Leung C. H.,Tang Y. Y., Tse K. W, Kwok P. C. K and Wong Y. K.,2003. Offline signature verification by tracking of feature and stroke position. Pattern Recognition, 2003, vol. 36, pp.91-101.

[17] Mahji B., Y. Santhosh Reddy. D. Prasanna Babu. 2006. Novel features for offline signature verification, International journal of Computers and Control, vol. 1, 2006, pp. 17-24. 